\title{
Onyx embolization of an intraosseous pseudoaneurysm of the middle meningeal artery in a patient with meningiomatosis, McCune-Albright syndrome, and gray platelet syndrome
}

\author{
Fabio Settecase, MD, MSc, FRCPC, Andrew D. Nicholson, MD, Matthew R. Amans, MD, MS, \\ Randall T. Higashida, MD, Van V. Halbach, MD, Daniel L. Cooke, MD, Christopher F. Dowd, MD, \\ and Steven W. Hetts, MD
}

Department of Radiology and Biomedical Imaging, University of California, San Francisco, California

A 13-year-old boy with meningiomatosis, McCune-Albright syndrome, and gray platelet syndrome presented with an enlarging "lump" on his right forehead. A head CT scan revealed a polyostotic fibrous dysplasia involving the entire skull. A 3.4-cm right frontal osseous cavity and an overlying right forehead subcutaneous soft-tissue mass were seen, measuring $5.2 \mathrm{~cm}$ in diameter and $1.6 \mathrm{~cm}$ thick. Ultrasound of the cavity and overlying mass showed swirling of blood and an arterialized waveform. MRI revealed an en plaque meningioma underlying the cavity. An intraosseous pseudoaneurysm fed by 3 distal anterior division branches of the right middle meningeal artery (MMA) with contrast extravasation was found on angiography. Two MMA feeders were embolized with Onyx, with anterograde filling of the intraosseous cavity with Onyx. A small pocket of residual intracavity contrast filling postembolization from a smaller third MMA feeder eventually thrombosed and the forehead lump regressed.

http://thejns.org/doi/abs/10.3171/2015.9.PEDS15267

KEY WORDS Onyx; endovascular; pseudoaneurysm; calvaria; McCune-Albright syndrome; gray platelet syndrome; meningioma; vascular disorders

\section{$\mathrm{M}$} IDDLE meningeal artery (MMA) aneurysms are very rare and most are traumatic in etiology. Twenty-seven cases of nontraumatic MMA aneurysms have been reported to date. , $, 2,5-7,12-20,22,24-30,34,35,37,38^{2}$ They may present with intracranial hemorrhage or may be discovered incidentally. Many MMA aneurysms have occurred in association with high-flow states, such as Paget's disease,, 24 dural arteriovenous fistulas and arteriovenous malformations, $, 13,14,27,29$ moyamoya disease, $,, 16,28$ and meningiomas. ${ }^{19,22,25}$ Others have occurred in the setting of a cavernous hemangioma of the skull, ${ }^{26}$ posterior cerebral artery occlusion,,$^{35}$ neurofibromatosis Type $2,,^{20}$ and after aneurysm coiling. ${ }^{17}$ In some cases, no associated disease process was found. ${ }^{6,30,37,38}$ Formation of an MMA aneurysm has not been previously described in the setting of McCune-Albright syndrome (MAS) or gray platelet syndrome (GPS). Both surgical and endovascular treatment of nontraumatic MMA aneurysms have been reported with success; however, the optimal management of these aneurysms is not established.

We describe the development of a pseudoaneurysm of the MMA with pseudoaneurysmal filling of an intraosseous cavity in the frontal bone of a patient with a multiplicity of potential etiological factors, including intracranial meningiomatosis, MAS, and GPS. We also describe successful treatment of the pseudoaneurysm using superselective Onyx-34 liquid embolic system embolization of feeding MMA branches as well as of the intraosseous cavity itself.

\section{Case Report}

History and Examination

A 13-year-old boy with a medical history of MAS, thrombocytopenia and GPS, meningiomatosis, recurrent epistaxis, and biliary atresia status after a failed Kasai 
(portoenterostomy) procedure, was brought into an outside primary care physician by his parents with a slowly enlarging "lump" on his right forehead. The mass had progressively increased in size over the previous 4 weeks. No antecedent trauma to the area was reported. He denied pain, tenderness, pressure sensation, or headaches. There had been no seizures, loss of consciousness, nausea, vomiting, or personality change. Because of GPS, the patient had also needed multiple platelet transfusions, especially in the setting of orthopedic surgery. Apart from an antibiotic course for recent pneumonia, the patient was not taking any medications, with the exception of having taken a bisphosphonate in the past. His cognitive development has been normal. He attends school and lives with his parents. Physical examination revealed short stature, limb deformities, and cherubic face characteristic of MAS. A 3-cm swollen raised area of skin was noted over the right forehead (Fig. 1A and B). The forehead lesion was soft, boggy, and nontender to palpation. A bruit was heard over the lesion on auscultation. The patient was neurologically intact.

At an outside hospital, an initial noncontrast head CT scan revealed a polyostotic fibrous dysplasia involving the entire skull. The CT scan also revealed a large right frontal osseous cavity with slightly irregular but well-defined borders within a thickened frontal bone, measuring $3.4 \times$ $3.0 \times 3.1 \mathrm{~cm}$ (Fig. 2). The density within the cavity measured 45 HU. A focal 3-mm dehiscence of the inner table and a $27-\mathrm{mm}$-wide defect in the outer table were noted. A large soft-tissue density mass was noted extending out of the right frontal osseous cavity into the overlying right
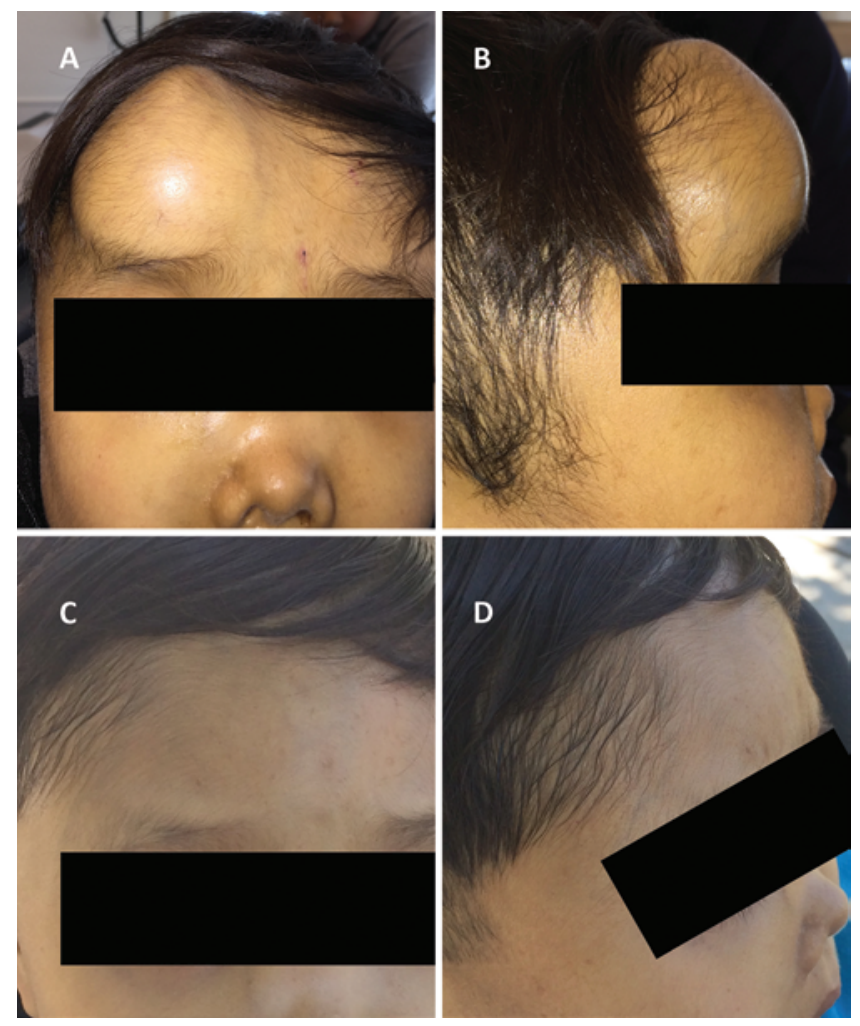

FIG. 1. Frontal (A) and lateral (B) photographs of the patient obtained immediately prior to embolization and 8 months after Onyx embolization (C) and (D). Figure is available in color online only. forehead subcutaneous tissue, measuring $5.2 \mathrm{~cm}$ in maximal diameter and $1.6 \mathrm{~cm}$ in thickness. Multiple smaller additional osseous cavities were seen throughout the skull and skull base. Ultrasonography of the right frontal mass was performed, which revealed swirling of blood within the mass with an arterialized Doppler waveform (Fig. 2). Because of the visualized flow, no biopsy was performed. Multiple meningiomas were also noted on head MRI, with an en plaque meningioma underlying the right frontal osseous cavity (Fig. 2).

The patient was transferred to our institution for further angiographic workup of the right frontal lesion and possible endovascular and/or neurosurgical treatment. On admission, the patient's platelet count was $21 \times 10^{9} / \mathrm{L}$. The patient underwent platelet transfusion to achieve a platelet count of $>50 \times 10^{9} / \mathrm{L}$.

A diagnostic cerebral angiogram revealed a right frontal intraosseous pseudoaneurysm fed by 3 distal frontal anterior division branches of the right MMA with extravasation of contrast into the intraosseous cavity (Fig. 3). No venous drainage from the cavity was seen. In addition, multifocal areas of beading likely representing tiny aneurysms/pseudoaneurysms and dysplastic changes were seen in multiple anterior division branches of the right MMA, including the branches feeding the pseudoaneurysm.

During angiography, significant left lung consolidation was noted in the absence of a left mainstem bronchus intubation. In addition, the anesthesia team noted a significant oxygen requirement. This was likely due to a chronic pneumonia, confirmed by the patient's parents. After consultation with our neurosurgical and pediatric neurology colleagues, the decision was made to treat the pseudoaneurysm endovascularly. The treatment was initially postponed to allow time for the patient's pneumonia to improve.

\section{Operation}

The forehead mass slowly enlarged over the following 8 days, and small focal areas of skin breakdown developed on the overlying scalp. Given the patient's history of thrombocytopenia and GPS, urgent endovascular embolization was performed. He received a transfusion of platelets to achieve a platelet count of $>75 \times 10^{9} / \mathrm{L}$. Written informed consent was obtained from the patient's parents for angiography and embolization. Under ultrasound guidance, a 5-F vascular sheath was placed in the left common femoral artery. A 5-F UCSF2 catheter (Codman) with a 0.035-in Bentson guidewire (Cook Medical) was used to select the right external carotid artery under fluoroscopic and roadmap guidance. Digital subtraction angiography (DSA) of the right external carotid artery confirmed the persistence of the right frontal intraosseous pseudoaneurysm with contrast extravasating into the cavity. No venous drainage was seen.

An eV3 Apollo microcatheter (eV3) was prepared with a Synchro-10 microwire. Under fluoroscopic and roadmap guidance, the microcatheter and microwire were used to selectively catheterize a distal frontal anterior division branch of the right MMA supplying the intraosseous pseudoaneurysm. After confirmation of the desired position and lack of anastomotic connections to the ophthalmic 

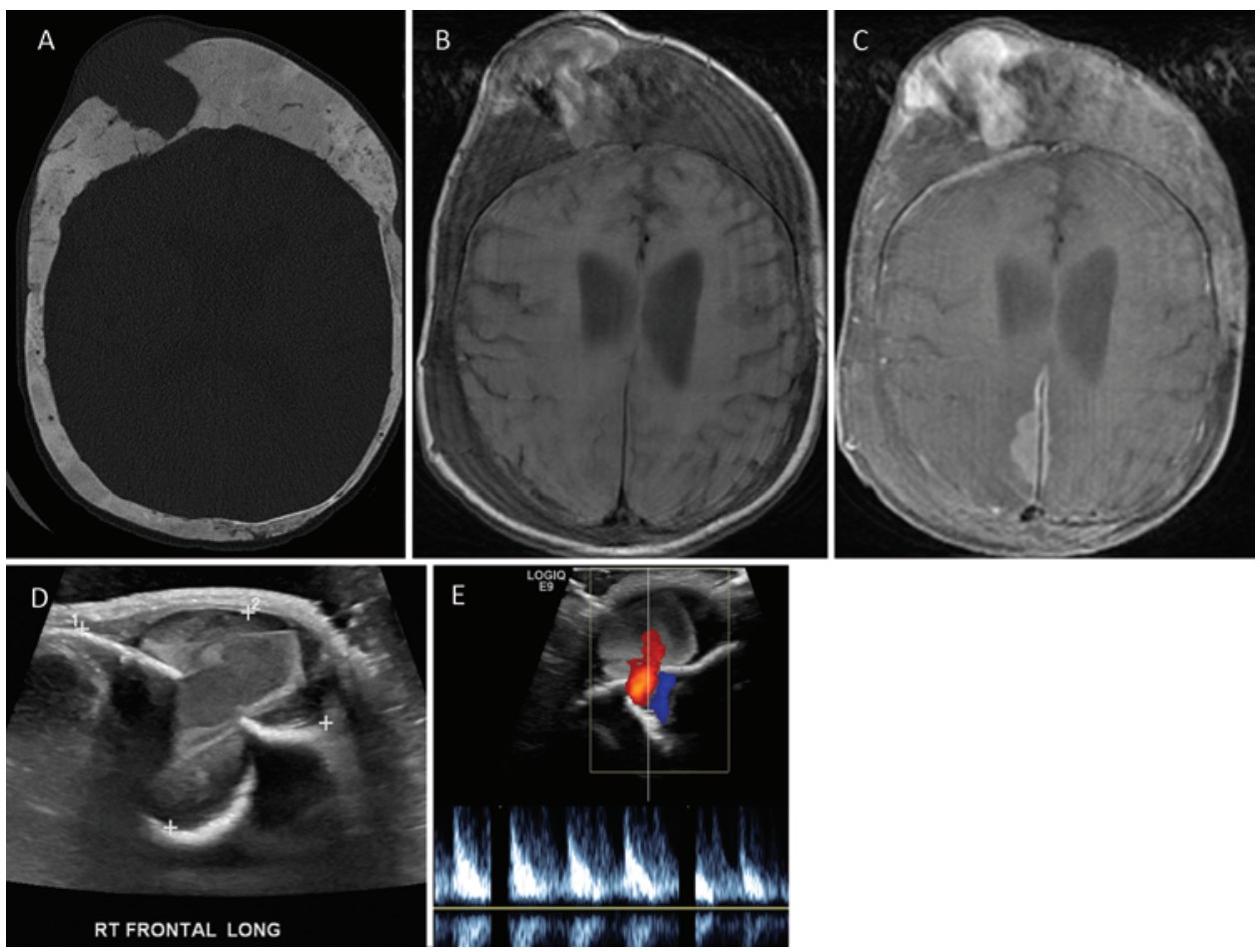

FIG. 2. A: Axial noncontrast CT bone window scan of the head demonstrating diffuse fibrous dysplasia of the skull characteristic of MAS. A large 3-cm intraosseous cavity with smooth margins is seen in the right frontal bone. Right overlying soft-tissue swelling is also present. B: Noncontrast T1-weighted MR image of the head demonstrating intrinsic T1 signal shortening in the right frontal osseous cavity, with associated pulsation artifact, concerning for hematoma and/or a vascular mass, such as a pseudoaneurysm. C: Postcontrast MR image of the head demonstrating dural thickening and enhancement in the right frontal region deep to the osseous cavity, likely representing an en plaque meningioma. An additional enhancing extraaxial mass along the right posterior falx is seen, also compatible with a meningioma. D: Gray scale ultrasound image of the right frontal extracranial softtissue component demonstrated swirling of isoechoic material within the cavity and extending into the subcutaneous soft tissue of the scalp. E: Color Doppler ultrasonography image demonstrating an arterial waveform within the lesion and the characteristic yin and yang sign of a pseudoaneurysm. Figure is available in color online only.

artery, the catheter was slowly flushed with $0.25 \mathrm{ml}$ of dimethyl sulfoxide. Under roadmap mask guidance, 4 vials of Onyx-34 (eV3) were used to embolize 2 of the 3 distal right frontal MMA branches supplying the pseudoaneurysm. Onyx was allowed to flow anterograde from MMA feeders and fill the intraosseous cavity dependently. The microcatheter was then slowly removed and the distal 1.5$\mathrm{mm}$ detachable tip of the Apollo catheter remained within the right MMA branch. Postembolization angiography demonstrated a small residual pocket of slow contrast filling in the deepest recess of the right frontal intraosseous cavity, supplied by the third right MMA branch. Several superficial temporal artery intraosseous collaterals from/ to this third MMA branch were noted. This small area of residual contrast filling within the cavity was believed to likely thrombose without further treatment because of its small size, slow filling, and persistence in the venous phase on angiography (indicating stasis of blood). In addition, superselective distal catheterization of the remaining MMA feeder was thought to be challenging anatomically. No further embolization was therefore undertaken, and the patient's received a platelet transfusion to maintain a platelet count $>75 \times 10^{9} / \mathrm{L}$ to augment thrombosis of the small residual cavity. There were no iatrogenic complications during the procedure. The patient was transferred to the pediatric intensive care unit for observation. He remained neurologically unchanged.

\section{Postoperative Course}

No further enlargement or bleeding of the right frontal mass was observed during the remainder of the hospitalization. Repeat ultrasonography with color Doppler interrogation performed at 24 and 72 hours after embolization demonstrated no residual flow within the right frontal lump and only a small pocket with residual arterialized flow in a deep pocket of the cavity near the inner table of the skull corresponding to the residual pocket seen at the conclusion of the embolization procedure. The patient received a platelet transfusion to keep his platelet count above $75 \times 10^{9} / \mathrm{L}$ for 48 hours. The patient's forehead mass, in the interim, had subjectively begun to regress. The patient was discharged on postprocedure Day 6 . In subsequent clinic follow-up, although the mother noted the forehead lump had markedly decreased in size over the weeks following the procedure, the forehead lump recurred and reached a similar size to the initial presentation 2 months later. A follow-up ultrasound at this time showed residual pseudoaneurysmal filling at the superolateral margin of the cavity. At 3 months after the initial Onyx embolization, repeat angiography was performed after 


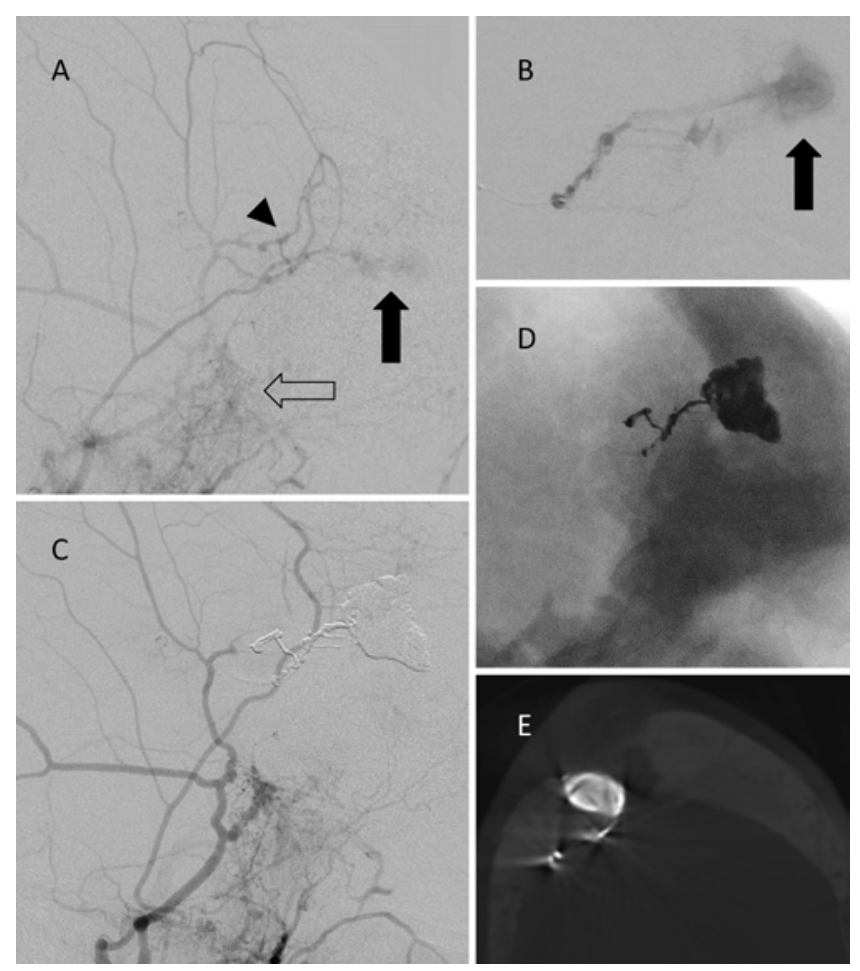

FIG. 3. Right external carotid artery injection, lateral view (A), demonstrating multiple dysplastic aneurysms (arrowhead) arising from distal anterior division frontal branches of the right MMA. Extravasation of contrast is seen into the right frontal osseous cavity (black arrow) arising from 3 separate distal MMA branches. In addition, several areas of osseous hypervascularity are seen related to fibrous dysplasia (white arrow). B: Superselective injection of one of the 3 right MMA feeding branches to the pseudoaneurysm again showing extravasation of contrast into the right frontal osseous cavity (arrow). Onyx embolization was initiated from this catheter position. C: Right external carotid artery injection, lateral view, obtained 3 months after Onyx embolization showing no residual pseudoaneurysm filling nor extravasation. $\mathrm{D}$ and $\mathrm{E}$ : Postembolization fluoroscopic image, lateral view (D), and axial DynaCT image (E) demonstrating Onyx filling the dependent portions of the osseous cavity and within distal right frontal MMA branches coursing along the dura.

preprocedure platelet transfusion to $>75 \times 10^{9} / \mathrm{L}$, which showed no residual pseudoaneurysm cavity filling or active extravasation, likely due to interval thrombosis (Fig. 3 ). No further platelet transfusions were performed the day after 3-month follow-up angiography. Four months after this last angiography session, clinical follow-up revealed that the forehead lump had completely resolved with no further recurrences (Fig. 1C and D).

\section{Discussion}

Aneurysms and pseudoaneurysms of the MMA are rare. Although usually caused by trauma, 27 cases of nontraumatic MMA aneurysms have been reported. ${ }^{1,2,5-7,12-20 \text {, }}$ 22,24-30,34,35,37,38 Most nontraumatic aneurysms have been reported with a variety of high-flow states, including Paget's disease, ${ }^{5,24}$ dural arteriovenous fistulas and arteriovenous malformations, $2,13,14,27,29$ moyamoya disease, ${ }^{7,16,28}$ and meningiomas. ${ }^{19,22,25}$ Others have occurred in the setting of a cavernous hemangioma of the skull, ${ }^{26}$ posterior cerebral artery occlusion,,$^{35}$ neurofibromatosis Type $2,{ }^{20}$ and after aneurysm coiling. ${ }^{17}$ In some cases, no associated disease process was found. ${ }^{6,30,37,38}$

The association of aneurysms with MAS has not been previously reported. MAS is a sporadic disease consisting of at least 2 of the following 3 features: polyostotic fibrous dysplasia, café au lait skin pigmentation, and/or autonomous endocrine hyperfunction (e.g., gonadotropinindependent precocious puberty, hyperthyroidism, acromegaly, or Cushing syndrome). ${ }^{3}$ The estimated prevalence of this disease is between 1 in 100,000 and 1 in 1,000,000 individuals with an embryonic somatic cell mutation that leads to the substitution of His or Cys for Arg at amino acid 201 of the $\mathrm{G}_{\mathrm{s}}$ alpha subunit, which stimulates cyclic adenosine monophosphate formation. This alteration has been observed in dysgenic endocrine and nonendocrine tissues from patients with MAS, but not in normal-appearing tissues, which is consistent with the mosaic distribution of abnormal cells. ${ }^{11,31,33,36}$ Various nonendocrine developmental abnormalities have been described in association with MAS, including congenital biliary atresia; gastrointestinal polyps; hyperplasia of the thymus, spleen, and pancreatic islet cells; microcephaly; and failure to thrive. ${ }^{33}$ There are only 2 case reports of meningiomas arising in association with MAS., ${ }^{4,8}$ A report of an orbital and palpebral arteriovenous malformation was described in a patient with MAS; 32 however, vascular anomalies are not associated features of MAS. There are several reports of aneurysmal bone cysts forming in the setting of fibrous dysplasia; ${ }^{10,21}$ however, the imaging and angiographic appearance in this case was not that of an aneurysmal bone cyst.

GPS is a rare inherited bleeding disorder characterized by thrombocytopenia and an absence of platelet $\alpha$ granules, resulting in typical gray platelets on peripheral smears. GPS is associated with a bleeding tendency, myelofibrosis, and splenomegaly. Although the underlying molecular basis is still not clear, the disease is caused by mutations in the NBEAL2 gene on chromosome 3. Cases of both autosomal-recessive and autosomal-dominant inheritance patterns exist. ${ }^{9}$ Although bleeding episodes are common in patients with GPS, aneurysm formation has not been previously reported in these patients.

Several predisposing factors likely contributed to the development of this patient's MMA intraosseous pseudoaneurysm. Dysplastic distal MMA branches feeding an en plaque meningioma were seen in the right frontal region on angiography. An association between nontraumatic MMA aneurysms and meningiomas may explain the initial formation of an aneurysm. The propensity for aneurysm rupture and inability to thrombose once ruptured in this patient were likely related to GPS and resultant thrombocytopenia. Once the aneurysm ruptured, softness of skull bones due to MAS likely facilitated growth of the pseudoaneurysm within an expanding osseous cavity via progressive scalloping. Once the outer table of the frontal bone was disrupted, the pseudoaneurysm extended into the overlying subcutaneous tissue, leading to the patient's presenting complaint. The multiple underlying diseases contributing to the formation of this patient's pseudoaneurysm are unique and have not been previously reported.

The natural history of nontraumatic MMA aneurysms is unclear because of their rarity. Although some aneu- 
rysms were found incidentally, several have presented with epidural, subdural, subarachnoid, intraparenchymal, or intraventricular hemorrhage..$^{15,16}$ Given the potential for intracranial hemorrhage or rehemorrhage, it is reasonable to prophylactically treat unruptured MMA aneurysms as well as those that have ruptured. In our case, the decision to treat urgently was based on the presence of an intraosseous pseudoaneurysm and resultant concern for eventual intracranial hemorrhage and/or percutaneous rupture in the setting of progressive enlargement of the aneurysm, GPS and thrombocytopenia, and overlying skin breakdown.

Of the 27 cases of nontraumatic MMA aneurysms that have been reported, approximately half of the patients underwent craniotomy and ligation, coagulation, or resection, $, 12,14,17,26-30,35,38$ while 6 were treated using endovascular techniques. ${ }^{16,19,20,22,25}$ Coil embolization was performed in 3 cases. ${ }^{16,19,25}$ In 2 cases the aneurysm and proximal MMA were embolized using $n$-butyl cyanoacrylate. ${ }^{20,22}$ While Onyx embolization of a traumatic MMA pseudoaneurysm has been reported, ${ }^{23}$ this is the first reported case of Onyx embolization of a nontraumatic MMA aneurysm. Use of $n$-butyl cyanoacrylate and Onyx promotes distal access compared with coils, as the agents can be delivered using smaller microcatheters, allowing for filling of both the aneurysm and the parent artery and thereby reducing the risk of aneurysm recanalization. The lower risk of nontarget embolization in the extracranial circulation also facilitates the use of liquid embolic agents. No established therapy exists, however, and the choice of surgical versus endovascular treatment of MMA aneurysms may be determined by other factors, such as the need for surgical evacuation of a hematoma. In our patient, given the size of the intraosseous pseudoaneurysm, presence of multiple MMA branch feeders, and the surgical risk posed by the presence of GPS and thrombocytopenia, we opted for embolization using a large volume of the Onyx liquid embolic system.

While perioperative transfusion of platelets facilitated initial thrombosis of the small residual cavity after Onyx embolization, GPS and chronic thrombocytopenia likely contributed to the recurrence of the lesion several weeks after embolization. The lesion "spontaneously" resolved after the next perioperative platelet transfusion at the time of the 3-month follow-up angiography session, likely due to thrombosis of the slow residual flow into the pseudoaneurysmal cavity seen at the end of the Onyx embolization. This highlights the importance of concomitant management of the underlying coagulopathy in the definitive treatment of this patient's pseudoaneurysm.

\section{References}

1. Akyol C, Turkoglu E, Kazanci B, Seckin H, Kertmen H, Bavbek M: Intraparenchymal haematoma related to true middle meningeal artery aneurysm: a case report. BMJ Case Rep 2009:bcr03.2009.1711, 2009

2. Akyuz M, Tuncer R: Multiple middle meningeal artery aneurysms associated with fistulous galenic arteriovenous malformation: a case report. Turk Neurosurg 20:544-546, 2010

3. Albright F, Butler AM, Hampton AO, Smith P: Syndrome characterized by osteitis fibrosa disseminata, areas of pigmentation and endocrine dysfunction, with precocious $\mathrm{pu}-$ berty in females. N Engl J Med 216:727-746, 1937
4. Bayas A, Naumann M, Wever S, Toyka KV: Meningioma associated with McCune-Albright syndrome. J Neurol 246:1199-1200, 1999

5. Berk ME: Aneurysm of the middle meningeal artery. Br J Radiol 34:667-668, 1961

6. Bollati A, Galli G, Gandolfini M, Orlandini A, Gualandi GF: True aneurysm of the middle meningeal artery. Case report. J Neurosurg Sci 24:89-92, 1980

7. Borota L, Marinkovic S, Bajic R, Kovacevic M: Intracranial aneurysms associated with moyamoya disease. Neurol Med Chir (Tokyo) 36:860-864, 1996

8. Fehlow P, Walther F, Assmann H: [McCune-Albright syndrome in association with meningioma and mental and psychological retardation.] Klin Padiatr 204:447-452, 1992 (Ger)

9. Gunay-Aygun M, Falik-Zaccai TC, Vilboux T, ZivonyElboum Y, Gumruk F, Cetin M, et al: NBEAL2 is mutated in gray platelet syndrome and is required for biogenesis of platelet $\alpha$-granules. Nat Genet 43:732-734, 2011

10. Haddad GF, Hambali F, Mufarrij A, Nassar A, Haddad FS: Concomitant fibrous dysplasia and aneurysmal bone cyst of the skull base. Case report and review of the literature. Pediatr Neurosurg 28:147-153, 1998

11. Happle R: The McCune-Albright syndrome: a lethal gene surviving by mosaicism. Clin Genet 29:321-324, 1986

12. Holland HW, Thomson JL: Aneurysm of the middle meningeal artery. Clin Radiol 16:334-338, 1965

13. Jin KH, Toyoda S, Kumagai K, Hashimoto T, Abe Y, Suzuki $\mathrm{K}$ : Non-traumatic middle meningeal aneurysm and angioma in a child. Brain Dev 3:323-328, 1981

14. Kähärä VJ: Middle meningeal artery aneurysm. Case illustration. J Neurosurg 91:518, 1999

15. Kobata H, Tanaka H, Tada Y, Nishihara K, Fujiwara A, Kuroiwa T: Intracerebral hematoma due to ruptured nontraumatic middle meningeal artery aneurysm-case report. Neurol Med Chir (Tokyo) 41:611-614, 2001

16. Koebbe CJ, Horowitz MB: A rare case of a ruptured middle meningeal aneurysm causing intracerebral hematoma in a patient with moyamoya disease. AJNR Am J Neuroradiol 25:574-576, 2004

17. Kohyama S, Kakehi Y, Yamane F, Ooigawa H, Kurita H, Ishihara S: Subdural and intracerebral hemorrhage caused by spontaneous bleeding in the middle meningeal artery after coil embolization of a cerebral aneurysm. J Stroke Cerebrovasc Dis 23:e433-e435, 2014

18. Korosue K, Kondoh T, Ishikawa Y, Nagao T, Tamaki N, Matsumoto S: Acute subdural hematoma associated with nontraumatic middle meningeal artery aneurysm: case report. Neurosurgery 22:411-413, 1988

19. Lama M, Mottolese C: Middle meningeal artery aneurysm associated with meningioma. J Neurosurg Sci 44:39-41, 2000

20. Lesley WS, Thomas MR, Abdulrauf SI: N-butylcyanoacrylate embolization of a middle meningeal artery aneurysm in a patient with neurofibromatosis type 2. AJNR Am J Neuroradiol 25:1414-1416, 2004

21. Lin WC, Wu HT, Wei CJ, Chang CY: Aneurysmal bone cyst arising from fibrous dysplasia of the frontal bone (2004:2b). Eur Radiol 14:930-932, 2004

22. Maekawa H, Tanaka M, Hadeishi H: Middle meningeal artery aneurysm associated with meningioma. Acta Neurochir (Wien) 151:1167-1168, 2009

23. Mehta S, Alawi A, Edgell R: Endovascular treatment of a traumatic middle meningeal artery pseudoaneurysm with Onyx LES: a case report and review of literature. J Neurointerv Surg 6 (Suppl 1):A73, 2014 (Abstract)

24. New PF: True aneurysm of the middle meningeal artery. Clin Radiol 16:236-240, 1965

25. O’Neill OR, Barnwell SL, Silver DJ: Middle meningeal 
artery aneurysm associated with meningioma: case report. Neurosurgery 36:396-398, 1995

26. Ohta H, Tanazawa T, Osuka K, Ito Y, Fukatsu T: True aneurysms of the middle meningeal artery associated with cavernous hemangioma of the skull-case report. Neurol Med Chir (Tokyo) 31:203-205, 1991

27. Onu DO, Hunn AW, Harle RA: A rare association of cerebral dural arteriovenous fistula with venous aneurysm and contralateral flow-related middle cerebral artery aneurysm. BMJ Case Rep 2013, 2013

28. Park YS, Suk JS, Kwon JT: Repeated rupture of a middle meningeal artery aneurysm in moyamoya disease. Case report. J Neurosurg 113:749-752, 2010

29. Sanchis JF, Orozco M, Cabanes J: Spontaneous extradural haematomas. J Neurol Neurosurg Psychiatry 38:577-580, 1975

30. Sandin JA III, Salamat MS, Baskaya M, Dempsey RJ: Intracerebral hemorrhage caused by the rupture of a nontraumatic middle meningeal artery aneurysm. Case report and review of the literature. J Neurosurg 90:951-954, 1999

31. Schwindinger WF, Francomano CA, Levine MA: Identification of a mutation in the gene encoding the alpha subunit of the stimulatory $\mathrm{G}$ protein of adenylyl cyclase in McCune-Albright syndrome. Proc Natl Acad Sci U S A 89:5152-5156, 1992

32. Scuderi G, Nucci C, Laghi A, Corsi A, Cerulli L: A rare case of McCune-Albright syndrome associated with glaucoma retinal degeneration and arteriovenous malformations. Eye (Lond) 10:752-755, 1996

33. Shenker A, Weinstein LS, Moran A, Pescovitz OH, Charest $\mathrm{NJ}$, Boney CM, et al: Severe endocrine and nonendocrine manifestations of the McCune-Albright syndrome associated with activating mutations of stimulatory G protein GS. J Pediatr 123:509-518, 1993

34. Takahashi M: Magnification angiography in moyamoya disease: new observations on collateral vessels. Radiology 136:379-386, 1980
35. Ushikoshi S, Houkin K, Itoh F, Saitoh H, Nozaki M, Kuroda $\mathrm{S}$, et al: Ruptured aneurysm of the middle meningeal artery associated with occlusion of the posterior cerebral artery. Case report. J Neurosurg 84:269-271, 1996

36. Weinstein LS, Shenker A, Gejman PV, Merino MJ, Friedman E, Spiegel AM: Activating mutations of the stimulatory G protein in the McCune-Albright syndrome. N Engl J Med 325:1688-1695, 1991

37. Zingesser LH, Schechter MM, Rayport M: Truths and untruths concerning the angiographic findings in extracerebal haematomas. Br J Radiol 38:835-847, 1965

38. Zubkov YN, Matsko DE, Pak VA: Saccular aneurysms of meningeal artery: case report. Neurosurgery 42:664-666, 1998

\section{Disclosures}

The authors report no conflict of interest concerning the materials or methods used in this study or the findings specified in this paper.

\section{Author Contributions}

Conception and design: Settecase, Hetts. Acquisition of data: Settecase, Nicholson, Hetts. Analysis and interpretation of data: all authors. Drafting the article: Settecase, Hetts. Critically revising the article: Settecase, Hetts. Reviewed submitted version of manuscript: all authors. Approved the final version of the manuscript on behalf of all authors: Settecase. Study supervision: Hetts.

\section{Correspondence}

Fabio Settecase, Department of Radiology and Biomedical Imaging, University of California, San Francisco, 505 Parnassus Ave., L351, San Francisco, CA 94143. email: fabio.settecase@ucsf.edu. 